\title{
Factors Influencing eHealth Literacy of Middle School Students in Korea: A Descriptive Cross-Sectional Study
}

\author{
Bu Kyung Park \\ College of Nursing, Research Institute of Nursing Science, Kyungpook National University, Daegu, Korea
}

Objectives: The aim of this study is to identify eHealth literacy level and to analyze the factors influencing eHealth literacy among middle school students in Korea. Methods: This descriptive cross-sectional study involved the administration of a self-report questionnaire. The participants were 799 middle school students. Descriptive statistics were analyzed via an independent $t$-test, Mann-Whitney $U$-test, and one-way ANOVA. Pearson correlation coefficients were used to test the correlations among variables. Multiple linear regression analysis was conducted to identify the factors influencing eHealth literacy. Results: eHealth literacy was substantially lower in students in their first year than in students in their third year of middle school. Participants with asthma, herniated disc, and atopic dermatitis had significantly lower eHealth literacy than those without the diseases. The major finding was that eHealth literacy of middle school students was influenced by their grade and number of diseases diagnosed. eHealth literacy was higher among students in their second and third years of middle school and among those who had fewer diagnosed diseases. Conclusions: eHealth literacy among adolescents is important for better health outcomes; however, middle school students with diseases had lower eHealth literacy than healthy students. Therefore, efforts to enhance eHealth literacy should focus on adolescents with diseases; moreover, health education in schools and healthcare settings for adolescents should include critical analytical skill development for better eHealth literacy.

Keywords: Health Literacy, Internet, Adolescent, Disease, Health Education

Submitted: May 27, 2019

Revised: 1st, July 17, 2019; 2nd, July 25, 2019

Accepted: July 25, 2019

\section{Corresponding Author}

Bu Kyung Park

College of Nursing, Research Institute of Nursing Science, Kyungpook National University, 680 Gukchaebosang-ro, Jung-gu, Daegu 41944, Korea. Tel: +82-53-420-4929, E-mail: bukpark@knu.ac.kr (https://orcid.org/0000-0001-6714-4226)

This is an Open Access article distributed under the terms of the Creative Commons Attribution Non-Commercial License (http://creativecommons.org/licenses/by$\mathrm{nc} / 4.0 /$ ) which permits unrestricted non-commercial use, distribution, and reproduction in any medium, provided the original work is properly cited.

(C) 2019 The Korean Society of Medical Informatics

\section{Introduction}

As a result of the broad access to the Internet and mobile devices, most of the population uses the Internet to search for health-related information [1]; health literacy via the Internet has thus become important. Health literacy is an essential skillset for improving health outcomes $[2,3]$. The eHealth Literacy Scale (eHEALS) that assesses eHealth literacy was developed based on the Lily model, which comprises six core types of literacy: traditional literacy, health literacy, information literacy, scientific literacy, media literacy, and computer literacy [4]. eHEALS has been used in multiple populations to measure the eHealth literacy of both younger and older adults [5-7], chronic patients [8], undergraduate students [9], 
and parents of children with certain diseases [10,11]. However, direct eHealth literacy level measurements among the younger population have been limited.

Adolescents are heavy Internet users; i.e., 98\% of adolescents reportedly use the Internet every day [12]. Developing eHealth literacy skills among adolescents is important because they consider the Internet their primary and best resource with which to find health information [13]. Previous studies have reported a significant relationship between Internet use and higher eHealth literacy levels among parents and adult patients $[5,11]$. In this light, the Internet promises to be an optimal medium for the dissemination of health information and the promotion of health among adolescents [14]. Therefore, eHealth literacy is warranted for using the Internet and it should therefore be measured for the adolescent population. However, few studies have focused on both the eHealth literacy of adolescents and the association between the health condition and eHealth literacy among this population.

There are many factors associated with eHealth literacy, including age, grade, chronic illness, perceived health, and frequency of hospital visits. For example, as basic literacy levels increase with age and grade among adolescents, so does the eHealth literacy capability to obtain health information from the Internet [4]. However, another study found that eHealth literacy was higher among younger and more-educated adults, which means that age is not associated with eHealth literacy, unlike education level [6]. Regarding health-related factors, people who were chronically ill had significantly lower eHealth literacy than people with no reported chronic illness [6]. Moreover, many chronic patients have insufficient capacity to search the Internet for information related to their disease [15]. Interestingly, perceived health was not a significant factor that affected eHealth literacy in that there was no significant difference between the high and low eHealth literacy groups in terms of perceived health [6]. There was an indirect effect path between eHealth literacy and the frequency of hospital visits that went via health-information-seeking behavior [16]. However, these studies targeted adult populations and there has been limited research on the factors affecting eHealth literacy among adolescents.

Considering that health literacy is one of the six core types of eHealth literacy, several studies have reported an association between health literacy and health outcomes. For example, the frequency of visits to the emergency department and hospitalization have a statistically significant relationship with the health literacy of the parents of children with special healthcare needs [17]. Meanwhile, low parental health literacy was independently associated with the perception of a child's health as poor and low health-related quality of life but was not associated with urgent care use [18]. Additionally, no significant differences in the health literacy of parents of asthma patients were found with regard to visits to the emergency department or number of hospital admissions [19]. Regarding glycemic control among parents of children with diabetes mellitus, a statistically significant relationship was observed between parent health literacy and glycemic control [20,21], whereas another study reported that there is no significant relationship between them [22]. Despite that parental health literacy has associations with health outcomes, there has been little research on health literacy among adolescents. Based on previous studies' findings, there are several gaps in the literature, suggesting that further research into 'direct assessment of child and adolescent health literacy' and 'assessment of the relationship between health literacy and healthcare utilization' is warranted [23]. This also corresponds with eHealth literacy; however, there is limited information regarding the assessment of a direct association between eHealth literacy and health-related variables.

The gap in the assessment and knowledge about the influence of factors associated with eHealth literacy among adolescents is particularly concerning and an important consideration for future research. Therefore, this study aimed at (1) identifying eHealth literacy levels, (2) examining the relationships between eHealth literacy and other factors, including general characteristics and diagnosed diseases, and (3) examining the effect of the factors on eHealth literacy among South Korean middle school students.

\section{Methods}

\section{Design}

This study is based on a descriptive cross-sectional design with a self-report questionnaire.

\section{Subjects}

A total of 799 participants were recruited using a convenience sampling method between January and March 2017 from two middle schools in Korea. These two schools were located in the Seoul metropolitan area. Participants were included if they (1) were currently attending middle school, (2) had obtained permission from their parents or legal guardian, and (3) understood the purpose of this study and agreed to participate in it. Fifteen participants were excluded due to missing data; thus, 784 participants were analyzed. Using the 
$\mathrm{G}^{\star}$ Power software program for a post-hoc analysis of multiple linear regression, the sample size of this study reached a power $(1-\beta)$ of $95 \%$ (effect size $=0.03, \alpha=0.05,9$ predictors) [24].

\section{Instruments}

The questionnaire contained information on general characteristics (sex, grade), the frequency of hospital admission, disease diagnosis, and score on the Korean version of eHealth Literacy Scale (K-eHEALS).

\section{1) eHEALS and K-eHEALS}

eHealth literacy is defined as the ability to read, use computers, search for information, understand health information, and put it into context [2]. A eHEALS was developed to access perceived skills at using the Internet for health-related information among a youth population aged 13-21 years [4]. Here, eHealth literacy was assessed using eHEALS. The scale comprises eight items rated on a 5-point Likert scale ranging from 1 (strongly disagree) to 5 (strongly agree). eHEALS has 10 items but items $\# 1$ and $\# 2$ are supplementary and are not included when calculating item mean scores.

K-eHEALS was developed and its reliability and validity were evaluated by healthy young adults from Korea as participants [25]. Cronbach's alpha of 0.88 was reported for KeHEALS [25], and here the Cronbach's alpha score was 0.96.

\section{Ethical Considerations}

The Institutional Review Board of Kyungpook National University approved this study (IRB No. 1041078-201611HRSB-212-01). Participants were given a detailed explanation of the study, including the purpose, process, and guarantees for anonymity. All participants provided written informed consent.

\section{Data Collection}

For data collection, the researcher explained the purpose, the importance, and the study process to the principal and the school health nurse of each middle school to obtain their cooperation. After obtaining permission from each middle school, a poster of this study was put up in the schools. If a student wanted to participate in the study, he or she had to visit the health room and receive the survey package.

For anonymity, one trained research assistant who was not related to the middle schools explained the purpose, importance, and process of the study to each participant and also provided and received the survey package. The survey package included written information on the study, a question- naire, written consent forms for parents and participants, and a return envelope.

The participants took the questionnaire home and put the completed questionnaire and consent into the return envelope. Participants were requested to fill out the eHealth Literacy Scale, and other information regarding health status (e.g., frequency of hospital admission, disease diagnosis) was requested from their parents for accuracy. Subsequently, the questionnaire was returned to the research assistant on another day. The questionnaire took approximately 15 minutes to complete. Participants received a small gift (a stationery set) as a token of appreciation for their participation.

Table 1. Characteristics of participants $(n=784)$

\begin{tabular}{lc}
\hline \multicolumn{1}{c}{ Characteristic } & Value \\
\hline Sex & $385(49.1)$ \\
Male & $399(50.9)$ \\
Female & \\
Grade & $235(30.0)$ \\
1 & $293(37.4)$ \\
2 & $256(32.7)$ \\
3 & $1.14 \pm 0.40$ \\
Frequency of hospital admission & $1.50 \pm 0.76$ \\
Number of diseases diagnosed & \\
Type of disease diagnosed & \\
Asthma & $760(97.0$ \\
No & $24(3.0)$ \\
Yes & \\
Gastrointestinal disease & $774(98.8)$ \\
No & $10(1.2)$ \\
Yes & \\
Scoliosis & $748(95.4)$ \\
No & $36(4.6)$ \\
Yes & \\
Herniated disc & $778(99.2)$ \\
No & $6(0.8)$ \\
Yes & \\
Atopic dermatitis & \\
No & \\
Yes & \\
Allergy (including food) & \\
No & \\
Yes & \\
\hline
\end{tabular}

Values are presented as number (\%) or mean \pm standard deviation. 


\section{Data Analysis}

Data were entered and analyzed using IBM SPSS Statistics version 23.0 for Windows (IBM Corp., Armonk, NY, USA). Descriptive statistics were used to define the participants' demographic characteristics and variables. Independent $t$ test, one-way ANOVA, and a post-hoc test (Scheffe test) were conducted to examine differences in eHealth literacy according to the sex and grade of participants. Mann-Whitney $U$-test was used when the assumptions of the $t$-test were not met. Pearson correlation coefficients were conducted to examine the association among frequency of hospital admission, number of diagnosed diseases, and eHealth literacy. Finally, multiple linear regression was used to examine all the variables that had a significant relationship with eHealth literacy via a univariate analysis and to determine the best determinants of eHealth literacy. The assumptions of the multiple linear regression analysis conducted via a residual analysis were generally met. A $p$-value of $\leq 0.05$ indicated statistical significance.

\section{Results}

\section{Participants Characteristics}

Half of the participants were males (49.1\%), and $37.4 \%$ were in the second year of middle school. The students had been admitted to a hospital 1.14 times (standard deviation [SD] $=0.40)$ and had been diagnosed with 1.50 diseases $(\mathrm{SD}=$ 0.76). With regard to the types of diseases diagnosed among the participants, $26.8 \%$ had atopic dermatitis, $9.5 \%$ had an allergy including a food allergy, and $4.6 \%$ had scoliosis (Table
1).

\section{2. eHealth Literacy among Middle School Students}

Descriptive statistics of eHealth literacy are shown in Table 2. Of the 10 items in eHEALS, participants reported the highest score for the importance of accessing health resources on the Internet ( $3.80 \pm 0.79$ of item \#2). On the other hand, the participants responded with the lowest score regarding where to find helpful health resources online $(3.26 \pm 0.83$ of item \#4). The item mean of eHEALS among middle school students was $3.59(\mathrm{SD}=0.71)$.

\section{Differences in eHealth Literacy according to General \\ Characteristics and Type of Disease Diagnosed}

Differences in eHealth literacy according to the general characteristics and type of disease diagnosed among participants are presented in Table 3. Statistical differences were found in students' grade $(F=6.470, p=0.002)$; the third-year students had significantly greater eHealth literacy than the first-year students. Additionally, a significant difference was also found in the types of disease diagnosed; for example, students with asthma had significantly lower eHealth literacy than those without asthma $(t=3.136, p=0.015)$. Furthermore, students with herniated disc (hereinafter, disc) and atopic dermatitis also had significantly lower eHealth literacy than students with no conditions $(t=1.910, p=0.047$ and $t=4.453, p=$ 0.001 , respectively).

Table 2. Descriptive statistics of eHEALS $(n=784)$

\begin{tabular}{lc}
\hline \multicolumn{1}{c}{ eHEALS } & Score (range 1-5) \\
\hline 1. How useful do you feel the Internet is in helping you make decisions about your health? & $3.62 \pm 0.81$ \\
2. How important is it for you to be able to access health resources on the Internet? & $3.80 \pm 0.79$ \\
3. I know what health resources are available on the Internet & $3.62 \pm 0.80$ \\
4. I know where to find helpful health resources on the Internet & $3.46 \pm 0.83$ \\
5. I know how to find helpful health resources on the Internet & $3.64 \pm 0.81$ \\
6. I know how to use the Internet to resolve my questions about health & $3.70 \pm 0.79$ \\
7. I know how to use health information I find on the Internet to help me & $3.68 \pm 0.79$ \\
8. I have the skills I need to evaluate the health resources I find on the Internet & $3.53 \pm 0.82$ \\
9. I can tell high-quality health resources from low-quality health resources on the Internet & $3.60 \pm 0.83$ \\
10. I feel confident in using information from the Internet to make health decisions & $3.51 \pm 0.84$ \\
Item mean (3-10 items) & $3.59 \pm 0.71$ \\
\hline
\end{tabular}

Values are presented as mean \pm standard deviation.

Questions \#1 and \#2 are recommended as supplementary items to be used with eHEALS to understand consumer's interest toward using eHealth in general. These items are not a formal part of the eHealth Literacy Scale, which comprises questions \#3-\#10. 
Table 3. Differences in eHealth literacy according to the general characteristics and type of diseases diagnosed $(n=784)$

\begin{tabular}{|c|c|c|c|c|}
\hline Characteristic & $\mathrm{n}$ & Score & $t$ or $F$ & $p$-value (Scheffe test) \\
\hline Sex & & & 0.202 & 0.840 \\
\hline Male & 385 & $3.60 \pm 0.74$ & & \\
\hline Female & 399 & $3.59 \pm 0.68$ & & \\
\hline Grade & & & 6.470 & 0.002 \\
\hline Third year & 256 & $3.70 \pm 0.78$ & & (3rd > 1st year) \\
\hline Second year & 293 & $3.62 \pm 0.69$ & & \\
\hline First year & 235 & $3.48 \pm 0.65$ & & \\
\hline \multicolumn{5}{|l|}{ Type of disease diagnosed } \\
\hline Asthma & & & 3.136 & $0.015^{\mathrm{a}}$ \\
\hline No & 760 & $3.60 \pm 0.71$ & & \\
\hline Yes & 24 & $3.31 \pm 0.43$ & & \\
\hline Gastrointestinal disease & & & 1.098 & $0.589^{\mathrm{a}}$ \\
\hline No & 774 & $3.59 \pm 0.70$ & & \\
\hline Yes & 10 & $3.33 \pm 1.07$ & & \\
\hline Scoliosis & & & 1.148 & $0.104^{\mathrm{a}}$ \\
\hline No & 748 & $3.60 \pm 0.70$ & & \\
\hline Yes & 36 & $3.46 \pm 0.81$ & & \\
\hline Herniated disc & & & 1.910 & $0.047^{\mathrm{a}}$ \\
\hline No & 778 & $3.59 \pm 0.70$ & & \\
\hline Yes & 6 & $3.04 \pm 1.21$ & & \\
\hline Atopic dermatitis & & & 4.453 & 0.001 \\
\hline No & 574 & $3.65 \pm 0.72$ & & \\
\hline Yes & 210 & $3.41 \pm 0.64$ & & \\
\hline Allergy (including food) & & & 1.244 & 0.216 \\
\hline No & 710 & $3.59 \pm 0.72$ & & \\
\hline Yes & 74 & $3.51 \pm 0.52$ & & \\
\hline
\end{tabular}

Values are presented as mean \pm standard deviation.

ann-Whitney $U$-test.

\section{Correlations and Regression Analysis with eHealth Literacy}

The number of hospital admissions $(r=-0.010, p=0.041)$ and number of diseases diagnosed $(r=-0.156, p=0.001)$ significantly correlated with eHealth literacy. Table 4 shows the factors associated with eHealth literacy among middle school students. Multi-collinearity between the predictors (e.g., numbers of hospital admissions, numbers of diseases diagnosed, asthma, disc, atopic dermatitis) was suspected but collinearity among these predictors was not found; for example, the range of tolerance, variation inflation factor, and condition index were $0.39-0.93$ (evaluation criteria: $>0.1$ ), 1.25-3.18 (evaluation criteria: <10), and 1.00-8.50 (evaluation criteria: $<30$ ), respectively.
To identify the critical factors that predict eHealth literacy, a multiple linear regression was conducted for independent variables, namely grade, number of hospital admissions, and number of diseases diagnosed as well as diagnoses of asthma, disc, and atopic dermatitis. The results showed that the prediction model for eHealth literacy was significant $(F=$ $6.570, p<0.001$ ), and the adjusted $\mathrm{R}^{2}$ of this model was 0.122 ; i.e., this model explained $12.2 \%$ of the variance of eHealth literacy. Moreover, third-year middle school grade $(\beta=0.137$, $p=0.001)$, second-year middle school grade $(\beta=0.091, p=$ $0.022)$, and the number of diseases diagnosed $(\beta=-0.159$, $p=0.001)$ were significant predictors of eHealth literacy among middle school students. These results indicated that eHealth literacy was higher when the students were in their 
Table 4. Factors influencing eHealth literacy among middle school students $(n=784)$

\begin{tabular}{lcccc}
\hline \multicolumn{1}{c}{ Variable } & B & Standard error & Standardized $\boldsymbol{\beta}$ & \multicolumn{1}{c}{$t(p$-value $)$} \\
\hline Constant & 3.529 & 0.086 & & $40.848(0.001)$ \\
Grade & & & 0.137 & $3.447(0.001)$ \\
$\quad$ Third year ${ }^{\mathrm{a}}$ & 0.215 & 0.062 & 0.091 & $2.290(0.022)$ \\
$\quad$ Second year ${ }^{\mathrm{a}}$ & 0.137 & 0.060 & 0.019 & $0.517(0.605)$ \\
Numbers of hospital admission & 0.033 & 0.064 & -0.159 & $-4.414(0.001)$ \\
Numbers of disease diagnosed & -0.152 & 0.034 & -0.015 & $-0.356(0.722)$ \\
Asthma & -0.060 & 0.169 & -0.129 & $-0.951(0.265)$ \\
Herniated disc & -0.122 & 0.135 & -0.088 & $-1.591(0.122)$ \\
Atopic dermatitis & -0.140 & 0.088 & & \\
\end{tabular}

${ }^{\mathrm{a}}$ Reference category: first year.

second and third years of middle school as well as when fewer diseases were diagnosed.

\section{Discussion}

To the best of the author's knowledge, this is the first research to focus on health-related factors influencing eHealth literacy among middle school students. Here, the findings revealed that grade, number of hospital admissions, and number of diseases diagnosed as well as diagnosis of asthma, disc, and atopic dermatitis were related to eHealth literacy. Specifically, second- and third-year middle school grade and number of diseases diagnosed appear to be the most important attributes of eHealth literacy.

A relatively higher score for eHealth literacy was reported (mean score, 3.59 of 5) in participants of the present study compared with the scores reported by previously published studies involving young adults in Korea (3.51 of 5) [25] and middle school students (3.44 of 5) [14]; however the score for the current participants was relatively lower than that for Hispanic adolescents in high schools (3.83 of 5) [26]. Considering the participants' age, they had adequate levels of eHealth literacy to use health information available on the Internet.

The most important and significant finding of this study was that eHealth literacy of middle school students decreased with an increasing number of diagnosed diseases. In other words, the health condition of adolescents was negatively associated with eHealth literacy. Unfortunately, no study has to date examined the relationship between eHealth literacy and number of diseases diagnosed among adolescents. Notably, a previously published study regarding the association between parents' eHealth literacy and child health [11] reported that parents having a child with good condition had significantly better eHealth literacy. In other words, the health condition of pediatric patients was negatively associated with their parents' eHealth literacy.

A possible reason for the parents of pediatric patients having better eHealth literacy whereas the patients had lower eHealth literacy was that the parents may actively search for information on their children's diseases using online sources. Evidence for such a prediction was found in previous studies reporting a significant relationship between higher frequency of Internet use with better eHealth literacy $[5,11]$. In contrast, when adolescents get sick, they may fail to attend school owing to a clinical appointment or may be unable to pay attention during lectures. This will lead to a lack of learning opportunities, thereby resulting in their low eHealth literacy level. A previous study also reported a significant association between parents' low health literacy and a higher number of days missed from school by the patients [17]; this may be linked to low eHealth literacy of patients. Therefore, healthcare providers and educators should pay attention to eHealth literacy levels among adolescents with any diagnosed disease as well as to their parents' eHealth literacy. The participants with asthma, disc, and atopic dermatitis in the present study showed significantly lower eHealth literacy than each of their counterparts. Children with Special Health Care Needs (CSHCN) have an increased risk of chronic physical, developmental, behavioral, or emotional conditions and thus they require more healthcare services than healthy children [27]; their diagnoses includes asthma, diabetes, etc. These children face numerous medical decisions throughout their lives, which prompts them to seek health information themselves. Considering high Internet usage by adolescents, eHealth literacy among CSHCN is a 
key indicator for the quality of self-management of chronic diseases; however, to the author's knowledge, to date, no studies have reported a direct examination of eHealth literacy among CSHCN. Therefore, efforts to improve eHealth literacy among CSHCN will be required for appropriate and effective utilization of online health information.

Here, there was no significant relationship between the number of hospital admissions and eHealth literacy. To date, there is limited information regarding the association between health care utilization and eHealth literacy of adolescents. However, a previous study reported that the parents of CSHCN with limited health literacy were more likely to visit the emergency department, with their children having a higher hospitalization frequency [11]. Thus, it is recommended that researchers examine the association of eHealth literacy of both parents and CSHCN with health care utilization frequency in future studies.

A previous study [9] reported that age, gender, grade point average (GPA), and frequency of Internet use were not significantly associated with eHealth literacy among university students (mean age, $21.9 \pm 3.48$ ). Another study [28] found that low health literacy was prevalent among higher grades among middle school students, with the third grade showing the lowest score of health literacy $(p=0.021)$. However, the results from this study showed that the second and third grades had significantly higher eHealth literacy than the first grade. Therefore, eHealth literacy might not have positive association with students' grade and there is a need for more research on this area.

A systematic review on health literacy among CSHCN [23] reported that parents' health literacy was associated with the patients' health outcomes, such as incidence of emergency department visits, hospitalization, and days missed from school [17]. In addition, parents' low health literacy was associated with decreased medication adherence of patients with glaucoma and diabetes management adherence among patients with type 1 diabetes [23]. Similarly, parents' eHealth literacy may have a significant relationship with health outcomes, similar to parents' health literacy. However, there is a lack of information on the relationship between parents' eHealth literacy and health outcomes and further assessment of this relationship.

Based on the present findings, healthcare providers should take into account that the eHealth literacy of pediatric patients may be lower than that of other healthy students even though they may have had more medical experience and faced numerous medical decisions throughout their lives. Efforts to improve their eHealth literacy should be consid- ered to provide a different direction of adolescents' health information-seeking behaviors.

Better eHealth literacy was associated with exposure to credible online health information sources (e.g., MedlinePlus) and increased likelihood of adequate health literacy [26]. Thus, health education in schools imparted to adolescents should include critical analytical skill development to enable them to search and reliably evaluate health information from credible online resources.

When healthcare providers meet adolescent patients, they should identify the adolescent patients having lower eHealth literacy level from the other healthy adolescents, so that they can provide those adolescents with the necessary health information in a manner that is easier to understand. This is important because, as the pressure to use the Internet to provide health information in healthcare setting increases, the relatively low eHealth literacy of the current tech-savvygeneration may be overlooked.

This study has several limitations. First, middle school students with low literacy levels might also have low eHealth literacy levels, but this study did not measure their literacy level. Research should be conducted where their literacy is controlled in order to determine the actual relationship between health-related variables and eHealth literacy. Second, the participants were recruited on a voluntary basis. This could have caused a selection bias toward middle school students with a higher interest in using the Internet. Third, this study recruited participants from only two middle schools in a single country. Thus, the sample may not be representative of the target population and may have potential limitations on the generalizability of the study results. Finally, this study did not assess Internet usage behaviors, age, and parents' eHealth literacy, which might have an influence on middle school students' eHealth literacy and health outcomes.

Despite these limitations, the findings of the present study will provide the literature with much needed regarding adolescent eHealth literacy and health-related variables.

In conclusion, this study is the first of its kind to describe eHealth literacy levels of middle school students and to investigate the association between their eHealth literacy and health-related variables. It is important to increase the low eHealth literacy level among middle school students with diseases to improve their health outcomes. The implications of this study are significant for school health educators and pediatric healthcare providers. This study revealed that eHealth literacy of middle school students was influenced by their grade and number of diagnosed diseases. The present findings suggest that efforts to enhance eHealth literacy 
should focus on adolescents with diseases, and to do so, health education in schools and healthcare settings should incorporate critical analytical skill development in adolescents.

\section{Conflict of Interest}

No potential conflict of interest relevant to this article was reported.

\section{Acknowledgments}

This research was supported by Kyungpook National University Research Fund in 2017.

\section{ORCID}

Bu Kyung Park (http://orcid.org/0000-0001-6714-4226)

\section{References}

1. Norman CD. Social media and health promotion. Glob Health Promot 2012;19(4):3-6.

2. Norman CD, Skinner HA. eHealth literacy: essential skills for consumer health in a networked world. J Med Internet Res 2006;8(2):e9.

3. Soellner R, Huber S, Reder M. The concept of eHealth literacy and its measurement. J Media Psychol 2014;26(1):29-38.

4. Norman CD, Skinner HA. eHEALS: the eHealth Literacy Scale. J Med Internet Res 2006;8(4):e27.

5. van der Vaart R, van Deursen AJ, Drossaert CH, Taal E, van Dijk JA, van de Laar MA. Does the eHealth Literacy Scale (eHEALS) measure what it intends to measure? Validation of a Dutch version of the eHEALS in two adult populations. J Med Internet Res 2011;13(4):e86.

6. Neter E, Brainin E. eHealth literacy: extending the digital divide to the realm of health information. J Med Internet Res 2012;14(1):e19.

7. Tennant B, Stellefson M, Dodd V, Chaney B, Chaney D, Paige S, et al. eHealth literacy and Web 2.0 health information seeking behaviors among baby boomers and older adults. J Med Internet Res 2015;17(3):e70.

8. Paige SR, Krieger JL, Stellefson M, Alber JM. eHealth literacy in chronic disease patients: an item response theory analysis of the eHealth literacy scale (eHEALS). Patient Educ Couns 2017;100(2):320-6.

9. Tubaishat A, Habiballah L. eHealth literacy among undergraduate nursing students. Nurse Educ Today 2016;42:47-52.

10. Manganello JA, Falisi AL, Roberts KJ, Smith KC, McKenzie LB. Pediatric injury information seeking for mothers with young children: the role of health literacy and ehealth literacy. J Commun Healthc 2016;9(3):223-31.

11. Knapp C, Madden V, Wang H, Sloyer P, Shenkman E. Internet use and eHealth literacy of low-income parents whose children have special health care needs. J Med Internet Res 2011;13(3):e75.

12. Pew Research Center. Internet/broadband fact sheet [Internet]. Washington (DC): Pew Research Center; 2019 [cited at 2019 Jul 6]; Available from: https://www. pewinternet.org/fact-sheet/internet-broadband/.

13. Manganello JA. Health literacy and adolescents: a framework and agenda for future research. Health Educ Res 2008;23(5):840-7.

14. Hove T, Paek HJ, Isaacson T. Using adolescent eHealth literacy to weigh trust in commercial web sites: the more children know, the tougher they are to persuade. J Advert Res 2011;51(3):524-37.

15. van der Vaart R, Drossaert $\mathrm{CH}$, de Heus M, Taal E, van de Laar MA. Measuring actual eHealth literacy among patients with rheumatic diseases: a qualitative analysis of problems encountered using Health 1.0 and Health 2.0 applications. J Med Internet Res 2013;15(2):e27.

16. Schulz PJ, Fitzpatrick MA, Hess A, Sudbury-Riley L, Hartung U. Effects of eHealth literacy on general practitioner consultations: a mediation analysis. J Med Internet Res 2017;19(5):e166.

17. DeWalt DA, Dilling MH, Rosenthal MS, Pignone MP. Low parental literacy is associated with worse asthma care measures in children. Ambul Pediatr 2007;7(1):2531.

18. Shone LP, Conn KM, Sanders L, Halterman JS. The role of parent health literacy among urban children with persistent asthma. Patient Educ Couns 2009;75(3):36875.

19. Wood MR, Price JH, Dake JA, Telljohann SK, Khuder SA. African American parents'/guardians' health literacy and self-efficacy and their child's level of asthma control. J Pediatr Nurs 2010;25(5):418-27.

20. Hassan K, Heptulla RA. Glycemic control in pediatric type 1 diabetes: role of caregiver literacy. Pediatrics 2010;125(5):e1104-8.

21. Pulgaron ER, Sanders LM, Patino-Fernandez AM, Wile D, Sanchez J, Rothman RL, et al. Glycemic control in young children with diabetes: the role of parental health 
literacy. Patient Educ Couns 2014;94(1):67-70.

22. Janisse HC, Naar-King S, Ellis D. Brief report: parent's health literacy among high-risk adolescents with insulin dependent diabetes. J Pediatr Psychol 2010;35(4):43640.

23. Keim-Malpass J, Letzkus LC, Kennedy C. Parent/caregiver health literacy among children with special health care needs: a systematic review of the literature. BMC Pediatr 2015;15:92.

24. Faul F, Erdfelder E, Buchner A, Lang AG. Statistical power analyses using $G^{\star}$ Power 3.1: tests for correlation and regression analyses. Behav Res Methods 2009;41(4):1149-60.

25. Chung S, Park BK, Nahm ES. The Korean eHealth Literacy Scale (K-eHEALS): reliability and validity testing in younger adults recruited online. J Med Internet Res 2018;20(4):e138.

26. Ghaddar SF, Valerio MA, Garcia CM, Hansen L. Adolescent health literacy: the importance of credible sources for online health information. J Sch Health 2012;82(1):28-36.

27. McPherson M, Arango P, Fox H, Lauver C, McManus $\mathrm{M}$, Newacheck PW, et al. A new definition of children with special health care needs. Pediatrics 1998;102(1 Pt 1):137-40.

28. Ran M, Peng L, Liu Q, Pender M, He F, Wang H. The association between quality of life(QOL) and health literacy among junior middle school students: a crosssectional study. BMC Public Health 2018;18(1):1183. 